\title{
Prospects for a Future JANET
}

\author{
Dr David Salmon ${ }^{1}$ \\ JANET(UK) \\ Lumen House, Library Avenue \\ Harwell Science and Innovation Campus \\ Didcot, Oxfordshire \\ OX11 OSG \\ UK \\ E-mail: David.Salmon@ja.net
}

\begin{abstract}
A brief overview of JANET and its transmission services is provided, noting those of particular relevance to Radio Astronomy and eVLBI. High transmission-rate operation at 40Gbit/s and a trial at 100Gbit/s are described. JANET Aurora, a dark-fibre facility supporting photonics and optical systems research is presented, leading to a view of technology options and potential new services which may be seen in the JANET network in 2013/14 following re-procurement and re-engineering.
\end{abstract}

\footnotetext{
$1 \quad$ Speaker
} 
Science and Technology of Long Baseline Real-Time Interferometry: The 8th International e-VLBI Workshop - EXPReS09

Madrid , Spain

June 22-26, 200 


\section{Introduction}

The backbone of JANET network has been running on a dedicated fibre infrastructure for about four years now, and when this was brought into service in 2006 it was the culmination of a three and a half year project beginning with a comprehensive requirements gathering phase within the JANET community followed by procurement, deployment and service migration periods. The network has been very successful, delivering both a high quality IP service and circuits (Lightpaths) to demanding communities and projects such as Radio Astronomers and eVLBI which are the focus of this workshop. Although there are still several years remaining within the operating contracts for the network, JANET(UK) is beginning to plan for the next version of the JANET network which is foreseen to be in service around 2013/2014.

This paper will give a brief summary of the current JANET network architecture and the transmission services it supports, followed by a discussion of some of main areas which will be investigated before the next procurement is started. These will range from contractual approaches to potential use of IRUs (Indefeasible Rights of Use) as vehicles for leasing fibres, and then moving on to engineering options at the optical transmission and circuit levels which are where we expect to see the most rapid evolution over the next two to three years.

\section{The JANET backbone}

The JANET fibre footprint currently uses about $6,500 \mathrm{~km}$ of leased fibre equipped with Ciena optical transmission systems. This provides the backbone of the JANET network which interconnects many regional aggregation networks to deliver services to the JANET community. The Universities and Colleges which form the Higher and Further Education organisations are connected by 17 of these regional networks to provide a resilient IP service which is the main JANET transmission service. In addition, it is possible to implement circuits (lightpaths) across the backbone between regional networks using dedicated wavelenghs, TDM based circuits, or packet/frame based circuits engineered with Ethernet/MPLS. This provides a flexible technology mix for supporting large projects and user communities with specific requirements for high-capacity point-to-point services, such as Radio Astronomy and eVLBI.

\section{Beyond 10Gb/s $-40 \mathrm{~Gb} / \mathrm{s}$ operations and $100 \mathrm{~Gb} / \mathrm{s}$ trials}

JANET(UK) undertook a trial of operating a $40 \mathrm{~Gb} / \mathrm{s}$ link on a separate fibre (not shared with any operational traffic) during 2008 using early production versions of Ciena transmission interfaces and Juniper router interfaces. This was successful and much of the JANET core network has now been upgraded to operate at $40 \mathrm{~Gb} / \mathrm{s}$ with second generation production interfaces. This was essential to maintain sufficient operational headroom on some of the core links. In fact, during the period when the trial was taking place, JANET-backbone traffic-levels increased to the extent that additional capacity was required, and extra $10 \mathrm{~Gb} / \mathrm{s}$ links were added to the core network to accomodate this growth, and bringing with them a more complex 
topology. The full $40 \mathrm{~Gb} / \mathrm{s}$ upgrade has enabled a reversion to the simpler topology with fewer links.

\subsection{Gb/s Operation}

Operation of the $40 \mathrm{~Gb} / \mathrm{s}$ network has been stable. The production transmission components deployed on the network use a more sophisticated modulation technique to encode multiple bits per symbol transmitted on the fibre, and also two independent sub-carriers with different polarisations. These schemes are more sensitive to Polarisation Mode Dispersion (PMD), and in some locations on the JANET backbone with older fibres it has been necessary to deploy Nortel equipment which has a modulation technique which accomodates a greater PMD per link.

\section{$3.2100 \mathrm{~Gb} / \mathrm{s}$ trials}

Even though $40 \mathrm{~Gb} / \mathrm{s}$ operation has been successful on JANET, traffic levels continue to grow and it is anticipated that JANET will need to deploy 100Gb/s links during 2010/2011 to accommodate these. A trial of $100 \mathrm{~Gb} / \mathrm{s}$ transmission was undertaken with Verizon Business and Nortel during early 2009. This deployed a pre-production Nortel $100 \mathrm{~Gb} / \mathrm{s}$ transmission system on a single wavelength within a fibre running between Reading and London. In order to confirm that this technique can operate without disrupting existing network traffic, two additional wavelengths were also configured to transmist traffic on channels immediately adjacent to the wave carrying the $100 \mathrm{~Gb} / \mathrm{s}$ traffic, and all wavelengths were operated at the standard $50 \mathrm{GHz}$ spacing. Simltaneous measurements of the bit-error rates on all three channels confimed that they were operating within normal margins without interfering with each other, and output from an optical spectrum analyser confirmed that the signal energy of the $100 \mathrm{~Gb} / \mathrm{s}$ wavelength was well contained in its allocated spectrum. An additional test using a PMD emulator inserted into the signal path demonstrated that the sophisticated modulation scheme adopted for the $10 \mathrm{~Gb} / \mathrm{s}$ transmisison system was the most tolerant to PMD. This shows that $100 \mathrm{~Gb} / \mathrm{s}$ transmission using this equipment should be very robust, and will be able to work reliably with older fibres, and across future networks which may contain significant numbers of optical switches.

\section{JANET Aurora}

JANET Aurora is a network of $850 \mathrm{~km}$ of dark fibre connecting five Universities in the UK and telehouse in London. It is a dedicated facility to support research into photonics and Optical systems and researchers have full access to the fibres for connecting their equipment without the constraints associated with full service infrastructures such as JANET and other similar networks. Results to-date include support of a Field Trial of Optical Time Division Multiplexing ${ }^{[1]}$, and the facility will also provide opportunities for application trials of emerging optical networking techniques. This work may inform the engineering approach to future versions of the JANET network, and efforts are being made to secure funding for the facility beyond 2011 when the current Aurora facilities contracts will terminate. 


\section{Futures for Radio Astronomy \& eVLBI on JANET}

High rate operations at $40 \mathrm{~Gb} / \mathrm{s}$ on JANET are now routine, and the positive outcome of the trials at $100 \mathrm{~Gb} / \mathrm{s}$ indicate that there should be no fundamental technical barrier at the network transmission level to implementing dedicated circuits (Lightpaths) for carrying high rate VLBI flows. However, NREN infrastructures usually operate within contractual frameworks with lifetimes of several years, and it may be some time before the majority of NRENs have had the opportunity to refresh their infrastructures to make such high rates possible within their networks. Such links are also likely to be expensive, so funding may be a significant issue in future, and individual NRENs are likely to have varying policies in this area, so these factors may make it challenging to establish international circuits at these rates.

\section{JANET Futures}

The JANET network will continue to evolve on the existing fibre infrastructure until late 2013 when the current contracts will end. The routing and transmission equipment will be upgraded as required to accommodate growing traffic levels, and it is expected that a significant number of $100 \mathrm{~Gb} / \mathrm{s}$ links will be operational at that point. The lightpath infrastructure will also be upgraded to meet demands, but no fundamental re-engineering is foreseen at this point.

However, new contracts and associated infrastructure will need to be in place during 2013 so that a managed service transition can be made. In preparation for this JANET(UK) has begun a project to look at both user requirements and technology options for this future JANET network, and this will study many areas from the fibres themselves through the transmission and routing/switching equipment, to legal options for structuring the contracts to leasing/accessing the fibres. The current contracts bundle both the fibre leases and the transmission equipment together, but decoupling these into separate agreements may offer advantages and this will be considered.

One of the major areas for examination will be optical transmission and possibilities for greater dynamism in services provision which may be possible with optical switching technologies such as Reconfigurable Optical Add-Drop Multiplexers (ROADMs) and Wavelength Selectable Switches (WSSs). These systems may also make it feasible to implement an "alien wave" service where third-party equipment would be allowed access to the optical multiplexers so that wavelengths originating in this equipment would then be transported across the network to their destination. This would require careful management of optical signal levels on the fibres, but could have potential advantages for projects requiring the capacities offered by whole wavelengths.

\section{References}

[1] G. Zarris, F. Parmigiani, E. Hugues-Salas, R. Weerasuriya, D. Hillerkuss, N. Amaya Gonzalez, M. Spyropoulou, P. Vorreau, R. Morais, S. K. Ibrahim, D. Klonidis, P. Petropoulos, A. D. Ellis, P. Monteiro, A. Tzanakaki, D. Richardson, I. Tomkos, R. Bonk, W. Freude, J. Leuthold, and D. Simeonidou, "Field Trial of WDM-OTDM Transmultiplexing Employing Photonic Switch Fabric- 
Based Buffer-less Bit-Interleaved Data Grooming and All-Optical Regeneration," in Optical Fiber Communication Conference, OSA Technical Digest (CD) (Optical Society of America, 2009), paper PDPC10. 